\title{
Comparison Analysis of the Effects of Small Resistor and Capacitor on Mitigation GIC in Power Grid
}

\author{
Yang peihong; Liu lianguang \\ State Key Laboratory of Alternate \\ Electrical Power System with Renewable Energy Sources \\ North China Electric Power University \\ Changping District, Beijing, China
}

\author{
Zheng xupeng; Feng shiwei; Li ya \\ School of Information Engineering \\ Inner Mongolia University of Science and Technology \\ Baotou, Inner Mongolia Autonomous Region, China
}

\begin{abstract}
Geomagnetically induced currents (GIC) can cause half cycle saturation of transformers and adversely affect the security operation of the power system, even result in large area blackouts. It may be possible to reduce the magnitude of GIC by installing small resistor or capacitor into transformers in a power grid. To compare the mitigation effect of the two schemes including small resistor and capacitor, four evaluation indexes used to assess the level of GIC are defined and the GIC level of $750 / 330 \mathrm{kV}$ power grid of Gansu province in China are evaluated considering five different mitigation configurations. It is found that the two schemes can decrease the magnitude of GIC relative to no installing mitigation device, but there is no obvious different between the two schemes to mitigate the GIC, and reduce the risk of GIC on power grid. By installing the small resistors or capacitor in $750 \mathrm{kV}$ substations, the mitigation effect is better than the $330 \mathrm{kV}$ substations.
\end{abstract}

Keywords-GIC; neutral point; power grid; mitigation scheme

\section{INTRODUCTION}

The severe disturbance of magnetic field raised by the solar storm toward earth, which caused severe disturbance of the geomagnetic field known as geomagnetic storm. The geomagnetic storm not only affects the satellite and communication, but also affects the ground power grid and the underground pipelines of oil and gas[1] .The impact and harm of geomagnetic storms on power grid have reached a consensus at home and abroad, its mechanism is the Geomagnetic Disturbance (GMD)of the Geomagnetic storms come into being the induced electric field, a earth surface potential (ESP)formed in this induced electric field generate the geomagnetically induced currents (GIC)in the circuits consisting of the transmission lines, power transformer windings, their neutral point, and the ground. The essence of GIC influence on power grid is derived from the transformer effect of GIC, secondary interference such as harmonic, temperature rise, vibration, noise and increase the loss of reactive power, which can threatening the safety of transformer and power grid when it's serious[3], and it has caused a large number of events of transmission line tripping, transformer broken and blackout accident happened[4]. Therefore, GIC mitigation has become a concern problem of many scholars at home and abroad.

In 1989, power grid in Quebec, Canada suffered a severe geomagnetic storm disaster; many foreign experts and scholars analyzed the accident later, and put forward a variety of the mitigation schemes of GIC and suggestions. In 1991, J.G.Kappenman put forward that partition/bypass device can be used in the transformer neutral point to reduce the GIC flow in the grid [5], and it was applied in the power grid in Quebec[6]. According to the actual situation of the power grid in Finland, R.Pirjola professor put forward install resistance in the transformer neutral point for GIC mitigation, and proved the feasibility of this scheme [7]. The mitigation of power grid GIC still in the exploration stage in China, it proposed the optimal configuration of the installing resistance in the neutral point of transformer can reduce influence of GIC to power grid [8], but that have not been verified in actual power grid. However, we do a lot of work on the mitigation of DC bias caused by the grounding current in the HVDC transmission system, the major mitigation measure is installing capacitor in and around the converter station transformer neutral point [9-10]. Though there are similarities between the DC bias phenomenon caused by HVDC transmission and geomagnetic storms, the grounding currents into the earth have smaller incidence than the GIC.

In this paper, we combined with the research achievements in GIC mitigation of both here and aboard, and we draw lessons from the dealing measures of DC magnetic bias caused by HVDC transmission grounding, using the $750 / 330 \mathrm{kV}$ power grids in Gansu province of china as the text case, we calculated and analyses the situation of GIC of power grids when the neutral point of transformer installed small resistor and capacitor, revealed the mechanism, performance and effect of these two mitigation schemes, which provides reference for grid electricity sector to carry out the mitigation and management work of GIC.

\section{GIC MODELING}

The effect of the induced electric field on the grid is equivalent to the voltage source connect between the different ground points, which are the integration along the line [11]:

$$
V_{i j}=\int_{i}^{j} \dot{E} \cdot d i
$$

where $\dot{E}$ is induction of the electric field vector which is related to earth stratification conductivity, geomagnetic field change rate and other factors. $i$ is line vector.

Define the vector $\mathbf{J}=\left[J_{1}, J_{2}, \cdots, J_{\mathrm{N}}\right)$, the $j$ th element is 


$$
J_{j}=\sum_{i=1, i \neq j}^{n} \frac{V_{i j}}{R_{i j}}
$$

where $\mathbf{J}$ represents the ground GIC current in the ideal case (ground branch resistance is 0 ), and $R_{i j}$ is DC resistance between any two nodes, the GIC current is expressed as

$$
\mathbf{I}_{\mathrm{GIC}}=(\mathbf{1}+\mathbf{Y Z Z})^{-1} \mathbf{J}
$$

where 1 represents unit matrix, $\mathbf{Y}$ represents admittance matrix, $\mathbf{Z}$ represents impedance grounding matrix.

On the basis of the above model, GIC calculation is carried out for 750 / 330kV power grid of Gansu and GIC distribution is analyzed for after installing the resistor and capacitor management device.

For the November 9-10, 2004 geomagnetic storms, the peak value of ground electric field in Xinjiang is $0.9397 \mathrm{~V} / \mathrm{km}$, Among, eastward component is $\mathrm{E}_{\mathrm{E}}=0.3026 \mathrm{~V} / \mathrm{km}$, Northward component is $\mathrm{E}_{\mathrm{N}}=-0.8896 \mathrm{~V} / \mathrm{km}[12]$. Since the magnetic storm is not the maximum storm of the 22th and 23th solar week, and the feature of the ionospheric current direction is not obvious in the middle and low latitude regions, and the geoelectric field in any direction may be occurred, the GIC of Gansu 750 / 330kV power grid can be calculated by the electric field of $1 \mathrm{~V} / \mathrm{km}$ to evaluate the GIC level, law and mitigation effect of GIC. The GIC values of the east / north to the electric field named as $\mathrm{E}_{\mathrm{E}} / \mathrm{E}_{\mathrm{N}}$ (denoted as $0^{\circ}$ and $90^{\circ}$ ) of any branch in the network at $1 \mathrm{~V} / \mathrm{km}$ are represented $\mathrm{a}$ and $\mathrm{b}$, when the amplitude of the electric field $\mathrm{E}$ in an arbitrary direction is $1 \mathrm{~V} / \mathrm{km}$, the GIC of the branch is given by

$$
I=a \cos \theta+b \sin \theta=A \cos (\theta-\alpha)
$$

where $A=(a 2+b 2) 1 / 2, \alpha=\arctan (b / a)$

From equation (4) we can see, when $\theta$ is equal to $\alpha$ or $\theta$ is equal to $\alpha \pm 180^{\circ}$, GIC reaches the maximum value which is represented $\mathrm{A}$.

In the case of Geoelectric field amplitude is unchanged, the maximum values and average value of the maximum of the neutral point GIC of the transformer in an arbitrary direction is shown as

$$
\begin{aligned}
I_{\max , \max } & =\max \left(\left|I_{j}\right|=\left|A_{j}\right| ; j=1,2, \ldots, M\right) \\
I_{\mathrm{avg}, \max } & =\frac{1}{M} \sum_{j=1}^{M}\left(\left|I_{j}\right| ; j=1,2, \ldots, M\right)
\end{aligned}
$$

where $\mathrm{M}$ is the number of transformers in the network.

Because it can be considered in China and other lowlatitude geoelectric fields in any direction may occur, that is, $1 \mathrm{~V} / \mathrm{km}$ of the geoelectric field in the $\left[1^{\circ} \sim 360^{\circ}\right]$ may occur. However, the GIC value of any branch in the network is symmetrical at $180^{\circ}$, in the calculation process only take $\left[1^{\circ} \sim 180^{\circ}\right]$.

For this reason, the maximum value of the average and the average value of the average of the neutral point of the transformer in the range of $\left[1^{\circ} \sim 180^{\circ}\right]$ of the earth electric field are as follows:

$$
\begin{aligned}
I_{\text {max avg }} & =\max \left(\frac{1}{M} \sum_{j=1}^{M}\left(\left|I_{\mathrm{GIC}_{n j}}\right| ; n=1,2, \ldots, K\right)\right) \\
I_{\text {avg, avg }} & =\frac{1}{K} \sum_{n=1}^{K}\left(\frac{1}{M} \sum_{j=1}^{M}\left(\left|I_{\mathrm{GIC} n j}\right| ; n=1,2, \ldots, K\right)\right)
\end{aligned}
$$

In the equations (7) and (8), $\mathrm{K}=180$

Formula (5) to (8) can be used as the four indicators to evaluate the power grid GIC level; it can also be used to compare small resistors and capacitors in the mitigation of power grid GIC effect on the reference.

\section{ANALYSIS OF THE Mitigation EFFECT}

In order to compare and analyze the mitigation effect on GIC in power grid by installing small resistor and capacitor installed in the neutral point, the $750 / 330 \mathrm{kV}$ power grid of Gansu province in China is employed as the study system, the geographical wiring diagram is shown in Fig. 1. It is assumed that all substation neutral points are operated by direct earthing, DC parameters of the whole network provided in [12].

\section{A. Parameters of Capacitor and Small Resistance}

Due to the neutral point of transformer installing capacitor has played an important role in completely isolate GIC, It is equivalent to break the neutral grounding point on the GIC calculation model, and there is no request for capacitors' parameters. The neutral point of transformer installing small resistor play a role in limiting GIC, and the small resistance will affect the effectiveness and reliability of transformer grounding. Therefore, neutral point of transformer installing small resistance need to meet the standard regulations of Design Specifications for AC Electric Device Grounding[13]. According to the actual situation of grounding resistance of the power grid substation in Gansu province, considering the maximum grounding resistance in the National standard, it is concluded that the resistance limit of small resistor installed in the $750 \mathrm{kV}$ substation in Gansu power grid is from $0.4 \Omega$ to $0.52 \Omega$, combined with the theoretical calculation, and the resistance limit of small resistances installed in the $330 \mathrm{kV}$ substation is from $0.5 \Omega$ to $0.7 \Omega$. In order to ensure the reliability of transformer grounding and the safety operation of power grid, this paper will take the fixed value of $0.4 / 0.5 \Omega$ as the limit of $750 / 330 \mathrm{kV}$ substation grounding resistance.

\section{B. Calculation of GIC Installing Capacitors/Small Resistors}

In order to compare the effect of two mitigation schemes, combined with the results of [2] and [13], five mitigation configurations are compared and analyzed, and the details are shown in table 1. 
TABLE I. DIFFERENT CONFIGURATIONS OF THE LOCATION OF NEUTRAL POINT SMALL RESISTOR AND CAPACITOR

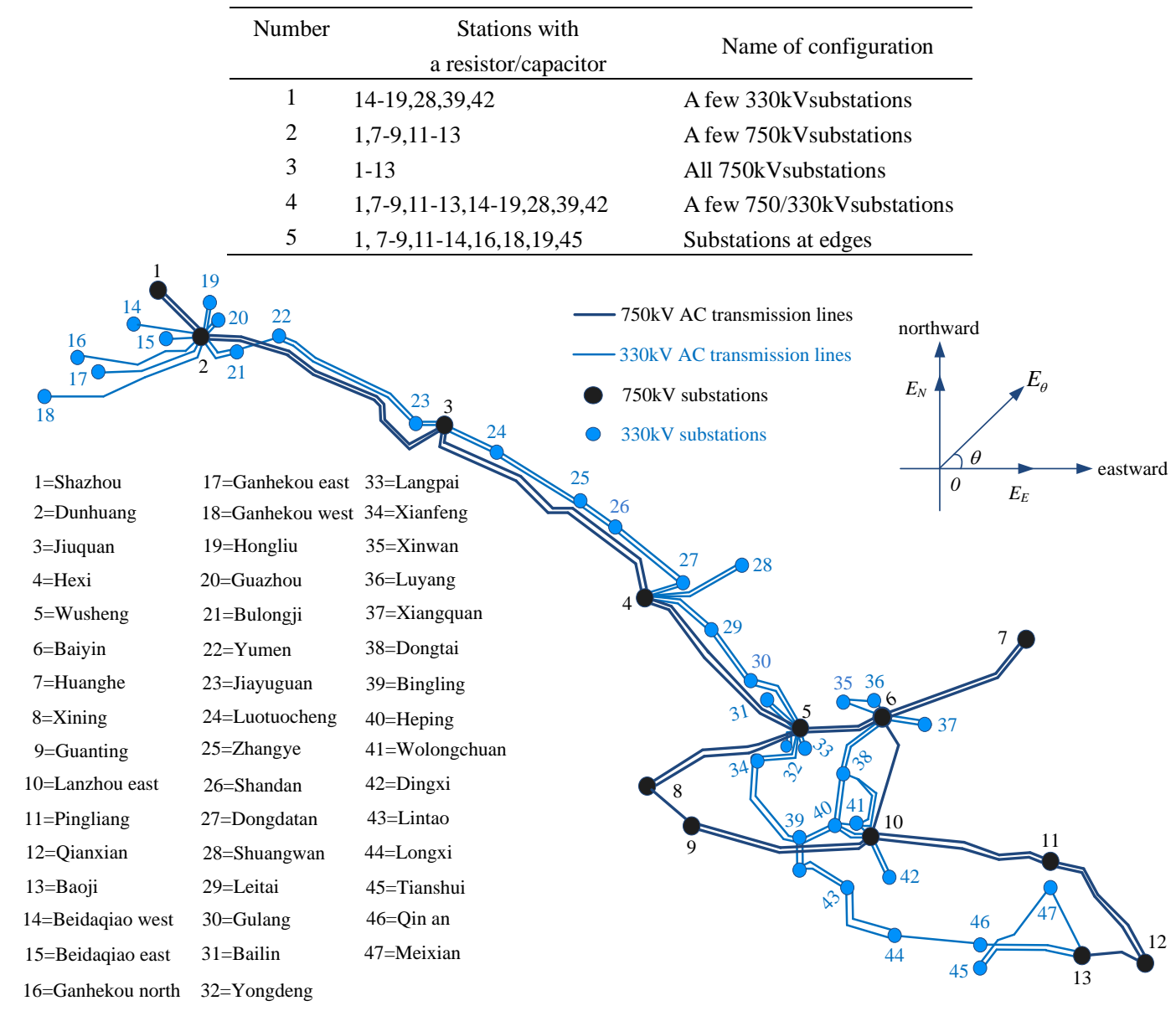

Fig. 1. Geographic wiring diagram of Gansu power grid in china

The GIC is calculated according to the five configurations defined in Table 1, taking the eastward earth electric field as
1V / km. The following Fig. 2 shows the substations with the neutral point GIC higher than 30A 
I(A)

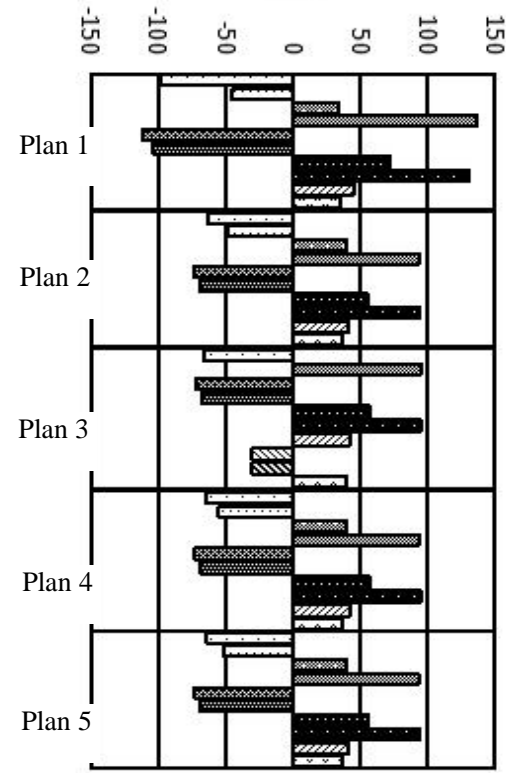

(a)
I(A)

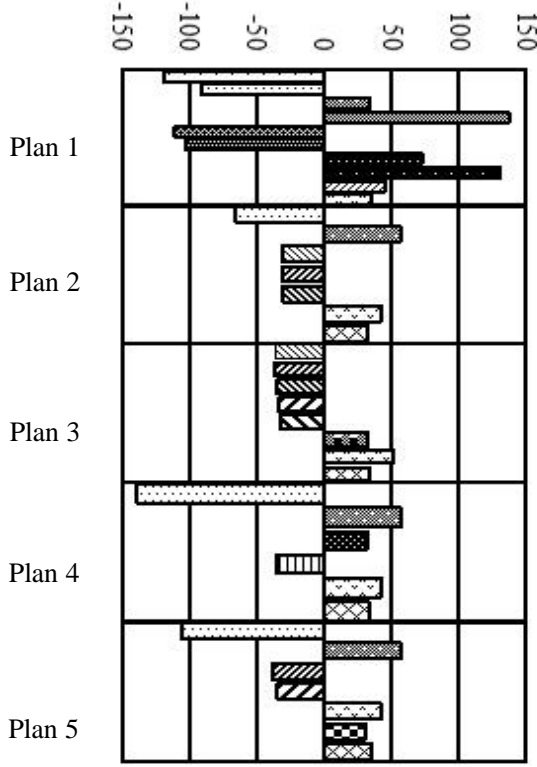

(b)

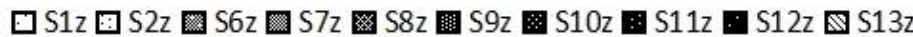

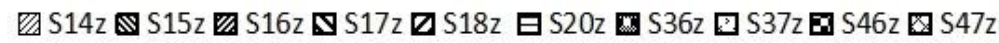

Fig. 2. Earthing GIC at individual stations of in case of small resistors (a) and capacitor(b) referring to five different configurations defined in Table

In Fig. 2, S1z represents the symbol of the neutral point of the transformer substation; the other symbols have the same meaning, where (a) and (b) are the calculation results when neutral point installing small resistors and capacitor
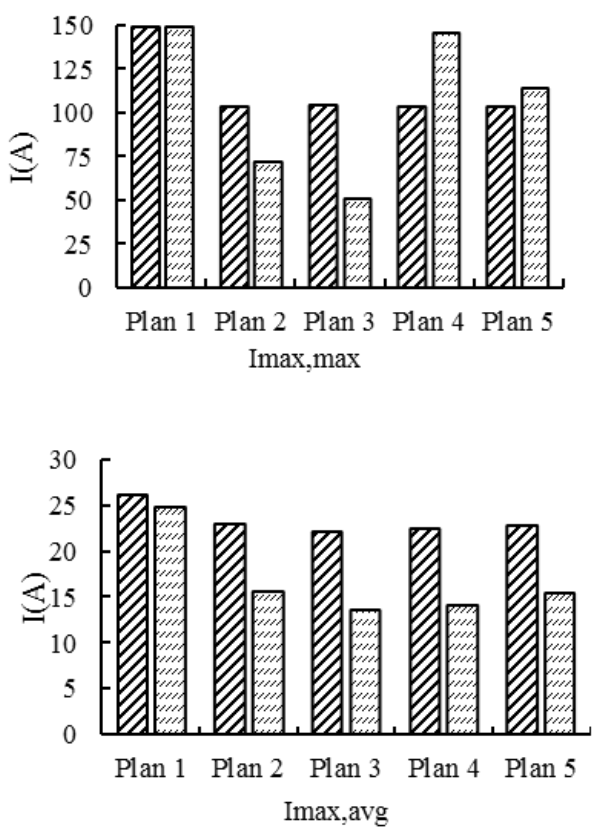

respectively. In order to compare adequately the effect of these two mitigation schemes, the GIC calculation is carried out by using the formulas (5) to (8). The results are shown in Fig. 3.
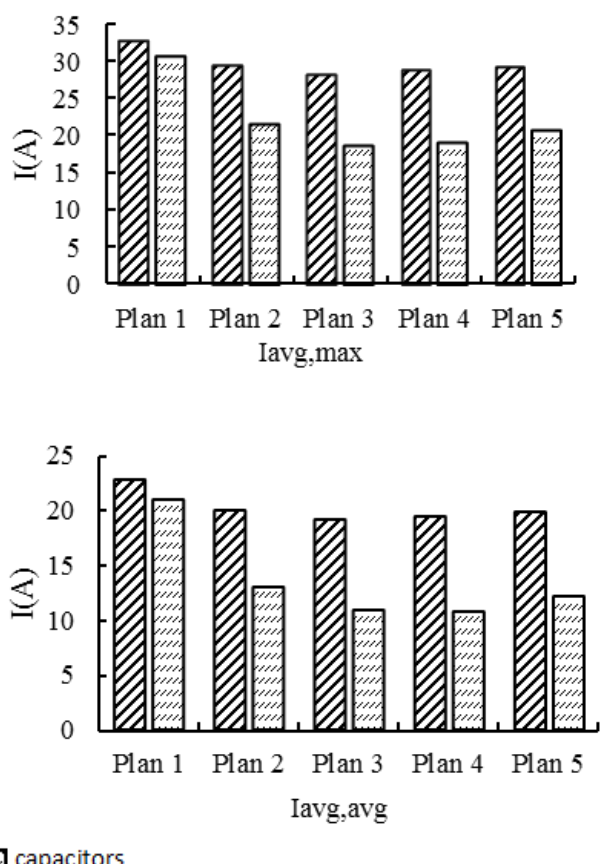

Fig. 3. Comparison of the absolute values of earthing GIC((Imax,max, Imax,max, Imax,max, Imax,max) referring to five different configurations defined in Table. 1 


\section{Analysis of Mitigation Effect}

Fig.2 point out installing resistor and capacitor in the mitigation GIC is very close in the scheme 1 , the maximum GIC of the installing resistor is 137.19A, and at the neutral point of the substation 7 , however, the maximum GIC of the installing capacitor is $137.41 \mathrm{~A}$, and also at the substation 7 neutral point; The maximum GIC of the installing resistor and capacitor at the substation 12 neutral point are $94.66 \mathrm{~A}$ and $65.33 \mathrm{~A}$ respectively in the scheme 2 ; Under the scheme 3 , the difference between the installing resistance and the capacitance is larger, Under low resistance, the maximum GIC value still occurs at the substation 12 and increases in contrast to the scheme 2, The main reason is that in all $750 \mathrm{kV}$ substation configuration of small resistors, it makes more difficult for GIC to circulate at the substations 4 and 5, and then be forced to circulate at substation 12 of the grid end, while the flow direction of GIC has also undergone great changes, This also shows that not mitigation effect in the more substation neutral point configuration of small resistor is better, but in the appropriate location of the configuration of small resistors is the best choice, Also in all $750 \mathrm{kV}$ substation installing capacitors, the maximum value of GIC is reduced to 50.88A, and the GIC high risk node is transferred to the substation 37 , the reason is that $750 \mathrm{kV}$ power grid has no GIC path, and then it transferred to the $330 \mathrm{kV}$ power grid, it makes GIC high-risk nodes transfer; Under the schemes 4 and 5, using small resistance effect is not obvious, which is similar to schemes 2 and 3 , while the use of capacitor mitigation, the GIC value of the high-risk node increases obviously.

Through comparative analysis of five programs, small resistors and capacitors have not shown a better mitigation effect, which shows that we have to consider a variety of factors to choose the most appropriate configuration methods and configuration location.

Fig. 4 gives more comprehensive information, In terms of $I_{\text {max,max }}$, we cannot compare the mitigation effect of the small resistance and capacitor, however, by the comparison

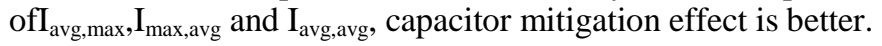

In summary, mitigation effect of the $750 \mathrm{kV}$ substation which installing small resistors and capacitors is better than the $330 \mathrm{kV}$ substation, especially in the $330 \mathrm{kV}$ substation installing capacitor will change the GIC distribution of the entire power grid, which makes the GIC of the high-risk node larger. Therefore, increase the resistance of configuring small resistors and a reasonable choice to configure. the locations which are particularly important to reduce the GIC of the power grid.

\section{CONCLUSION}

For the feasibility of power grid GIC can be mitigated by connecting small resistor or capacitor in the transformer neutral point, the control effect of the above two measures is analyzed and compared. GIC calculation results of $750 / 330 \mathrm{kV}$ power grid in Gansu Province of China show that as a result of the mechanisms of small resistors and capacitors in the mitigation of power grid GIC are different, resulting in mitigation effect of them under the same program is quite different, which cannot explain what a better mitigation. In the $750 \mathrm{kV}$ transformer neutral point connecting small resistors or capacitors for the GIC mitigation effect of the entire power grid is better than $330 \mathrm{kV}$ transformer, and then it can be drawn the conclusions that in the highest voltage level power grid control GIC better.

\section{REFERENCES}

[1] R.Pirjola. Geomagnetically induced currents during magnetic storms [J]. IEEE Trans. Plasma Sci, 2000, 28(6):1867-1873.

[2] Liu L G, Wang K P, Guo S X, et al. Characteristics of GIC interaction in a dual-voltage-level power network[J]. Sci Sin Tech.2015, 45:13111320.

[3] Wen Jun , Liu Lianguang, Xiang Song, et al. Influences of geomagnetic induced currents on security and stability of power systems[J] . Power System Technology, 2010, 34(11):24-30(in Chinese).

[4] Wik M, Viljanen A, Pirjola R, et al. Calculation of geomagnetically induced currents in the $400 \mathrm{kV}$ power grid in southern Sweden[J]. Space Weather, 2008, 6:S07005.

[5] KappenmanJG, NorrSR, SweezyGA . GIC Mitigation:A neutral blocking/bypass device prevent the flow of GIC in power system[J]. IEEE Transactions on power Delivery, 1991, 6(3):12711283.

[6] Leonard Bolduc, Michel Granger, Gregoire Pare. Development of a DC Current-Blocking Device for transformer neutrals[J] IEEE Transactions on power Delivery, 2005, 20(1):163-168.

[7] Risto Pirjola. Averages of geomagnetically induced currents (GIC) in the finish $400 \mathrm{kV}$ electric power transmission system and the effect of neutral point reactors on GIC [J]. Journal of atmospheric and solarterrestrial physics, 2005, 67:701-708.

[8] Wu weili, Liu lianliang. An optimal control method for overcoming clustering transformer reactive power losses due to geomagnetic strom[J].Automation of electric power system, 2014, 38(2):32-37.

[9] Wang Huawei, Lin Shaobo, Wang Zuli, et a1. Problems analysis and improvement for neutral DC current blocking device used in Xizhe UHVDC [J]. Power System Technology, 2015, 39(6):1600-1604.

[10] Yu Yongjun, Yang Qi, Hou Zhiyuan, et al. Influence of grounding current in UHVDC transmission project from Tianshan to Zhongzhou on Hami AC power system in Xinjiang uygur autonomous region[J]. Power System Technology, 2014, 38(8):2298-2303.

[11] Boteler D H, Pirjola R J, Nevanlinna H. The effects of geomagnetic disturbances on electrical systems at the Earth surface[J]. Advances Space Research, 1998, Vol.22: 17-27.

[12] Guo shixiao.Interaction and impact of geomagnetically induced current in different voltage level power systems[D].North China Electrical Power University,2015.

[13] China Electricity Council. GB50065-2011. design specifications for AC electric device grounding. Beijing: China Plan Publishing Company, 2011 . 